\title{
Tumor microenvironment and future targets of immunotherapy in breast cancer
}

\author{
Qi Wang, Qiang Liu \\ Breast Tumor Center, Sun Yat-Sen Memorial Hospital, Sun Yat-sen University, Guangzhou 510120, China \\ Contributions: (I) Conception and design: None; (II) Administrative support: None; (III) Provision of study materials or patients: None; (IV) \\ Collection and assembly of data: None; (V) Data analysis and interpretation: None; (VI) Manuscript writing: All authors; (VII) Final approval of \\ manuscript: All authors. \\ Correspondence to: Qiang Liu. Breast Tumor Center, Sun Yat-sen Memorial Hospital, Sun Yat-sen University, 107 Yanjiang West Road, Guangzhou \\ 510120, China. Email: victorlq@hotmail.com.
}

\begin{abstract}
Tumor microenvironment (TME) comprises many non-malignant cells that act as accomplices to support tumor. Both immune cells and stromal cells in TME play an essential role in regulating many aspects of a tumor, including tumor growth, metastasis and drug resistance. The complex interactions between malignant cells and non-malignant cells in the TME determine the fate of a tumor. A comprehensive understanding of the components of TME will help us to choose the right target for cancer therapy, especially immunotherapy. In this review, we discuss the phenotypes, functions and potential therapeutic value of various cellular components in the TME.
\end{abstract}

Keywords: Breast cancer; tumor microenvironment (TME); immunotherapy; stromal cell; T cell; B cell; myeloidderived suppressor cells (MDSCs)

Received: 17 March 2020; Accepted: 30 March 2020; Published: 10 April 2020

doi: $10.21037 /$ tbcr.2020.03.07

View this article at: http://dx.doi.org/10.21037/tbcr.2020.03.07

\section{Introduction}

Breast cancer is the most common type of cancer in women worldwide. The treatment of breast cancer has evolved significantly over the past decades, with the five-year survival rate increased to $80-90 \%$ in most countries today. Although breast cancer has a relatively good prognosis, it remains the leading cause of cancer mortality worldwide. All the current treatments, including surgery, chemotherapy, endocrine therapy, radiation therapy and targeted therapy, are targeting cancer cells and reach a plateau that is difficult to improve.

Cancer is not only formed by malignant cancer cells, but also comprised of many other non-malignant cells in the tumor microenvironment (TME) that behave as accomplices to support tumor growth. The strategy to target cancer cells alone has been shown to be ineffective in many cancer patients, especially in metastatic breast cancer patients that are incurable and inevitably become resistant to traditional treatments sooner or later. Cancer immunotherapy becomes a very hot topic in recent years, with promising results in melanoma and lung cancer. Different from most cancer treatments that directly target cancer cells, immunotherapy targets the immune cells in TME and only indirectly targets cancer cells by enhanced immunity against tumor. Immunotherapy may be a better strategy than traditional cancer cell-targeting treatments because cancer cells are very cunning and adaptable to develop resistance.

TME, the milieu cancer cells reside and grow, plays an important role in regulating the initiation, progression and metastasis of a tumor. TME has commanded the attention of researchers from early years $(1,2)$. TME is a dynamic mixture of a variety of cells, including immune cells, non-immune cells, as well as multiple modules including cytokines, growth factors and enzymes (3). The immune cells, stromal cells and other components in TME interact with each other to determine whether foster or restrain 
cancer cells. It is believed that TME is anti-tumor and helps to eliminate cancer cells at early stages of tumorigenesis. However, as the tumors progress and become hard to kill, TME is changed by malignant cells and turns to pro-tumor at later stages of tumorigenesis. Therefore, targeting the TME components that are accomplices of cancer cells will significantly improve the therapeutic response and the prognosis of cancer patients.

However, the current understanding of TME is still poor and the immunotherapy in solid tumors has only shown limited success, with anti-PD-1/PD-L1 antibodies being the only clinically available treatments in advanced breast cancer so far. Moreover, the biomarkers for breast cancer patients are not well established. It is crucial to better understand the complexity and diversity of TME and its influence on treatment response to move immunotherapy forward. In this review, we will introduce the cellular components of the TME and their function to present a comprehensive picture of TME and future directions of immunotherapy.

\section{Cellular components in the TME}

Cellular components in the TME vary significantly, both in the number and phenotype of immune and stromal cells. They play a pivotal role in either promoting or suppressing cancer. Different cells are recruited into the tumor by multiple regulatory modules and come into play.

\section{Immune cells}

Immune cells in TME consist of innate immune cells and adaptive immune cells. Innate immune cells mainly include tumor-associated macrophages (TAMs), myeloid-derived suppressor cells (MDSCs), dendritic cells (DCs), natural killer cells (NK cells), tumor-associated neutrophils (TAN), and mast cells. Adaptive immune cells are mainly comprised of $\mathrm{T}$ cells and $\mathrm{B}$ cells. The types, markers, classifications and functions of immune cells in TME are summarized in Table 1.

The immune cells in TME communicate with each other by cell-to-cell contact or secretion of cytokines and chemokines. Given their diversity, it is difficult to use a few markers to illustrate these cells in a comprehensive way. Based on gene expression profile data from bulk tumor tissues, several techniques including CIBERSORT (4) and XCell (5) can be used to estimate the abundance of immune cells infiltrating into tumor. These methods can provide the heterogeneity of immune cell composition in complex tissues, but lack cell proportion, spatial distribution and function. Mikhail Binnewies et al. (6) proposed a classification system to divide the tumor immune microenvironment (TIME) into three categories: infiltrated-excluded (I-E) TIMEs, infiltrated-inflamed (I-I) TIMEs, and tertiary lymphoid structures (TLS)TIMEs. Cytotoxic lymphocytes (CTLs) in I-E TIMEs are localized along the border of tumor or caught in the fibrotic nest, seemingly "excluded" from infiltrating into tumor. In contrast, I-I TIMEs have high infiltration of PD1-expressing CTLs. TLS-TIMEs, a subclass of I-I TIMEs, have immune cell components similar to lymph nodes. Moreover, tumors having I-E TIMEs are thought to be immunologically "cold", whereas tumors with I-I TIMEs are considered as immunologically "hot". This classification can help us understand the TME more thoroughly and facilitate the treatment decision.

TAMs are the most abundant immune cells in TME (7). It is reported that macrophages have two functional phenotypes, M1 $\left(\mathrm{CD} 40^{+}\right)$and M2 $\left(\mathrm{CD} 169^{+} \mathrm{CD} 206^{+}\right)$ macrophages (8). M1 macrophages, which are activated by IFN- $\gamma$ or LPS to secret pro-inflammatory cytokines (IL-1 $\beta$, IL-6 and TNF- $\alpha$ ), are considered anti-tumor. Conversely, M2 macrophages, which are induced by IL- 4 or IL-13 to secrete immunosuppressive factors like IL-10 and TGF- $\beta$, are pro-tumoral. In fact, most TMEs have M2 macrophages that promote angiogenesis and tissue remodeling. However, M1 and M2 phenotypes are two extreme polarization of TAMs. TAMs can exist in a continuous spectrum between M1 and M2 status, and the status of TAMs can be flexible and reversible. Therefore, switching the phenotype of macrophages from pro-tumor to anti-tumor could be an excellent way to change TIME for therapeutic strategies (9). For instance, it was shown that M2 macrophages had high phosphoglycerate dehydrogenase (PHGDH) activity to promote M2 polarization and supports macrophage proliferation, thus PHGDH could be a promising metabolic checkpoint to fine-tune macrophages against cancer (10).

Myeloid-derived suppressor cells (MDSCs) are a group of myeloid cells with a potent immunosuppressive capacity that distinct them from other myeloid cells like monocytes and neutrophils (11). Based on their phenotypic and morphological features, MDSCs have two major subsets: polymorphonuclear-MDSCs (PMN-MDSCs) defined as $\mathrm{CD} 11 \mathrm{~b}^{+} \mathrm{CD} 14^{-} \mathrm{CD} 15^{+}$or $\mathrm{CD} 11^{+} \mathrm{CD} 14^{-} \mathrm{CD} 66 \mathrm{~b}^{+}$, and monocytic-MDSCs (M-MDSCs) as CD11 $\mathrm{b}^{+} \mathrm{CD}_{1} 4^{+} \mathrm{HLA}^{-}$ $\mathrm{DR}^{-h_{0}} \mathrm{CD} 15^{-}$(12). MDSCs will not appear in normal tissue, 
Table 1 Immune cells in TME

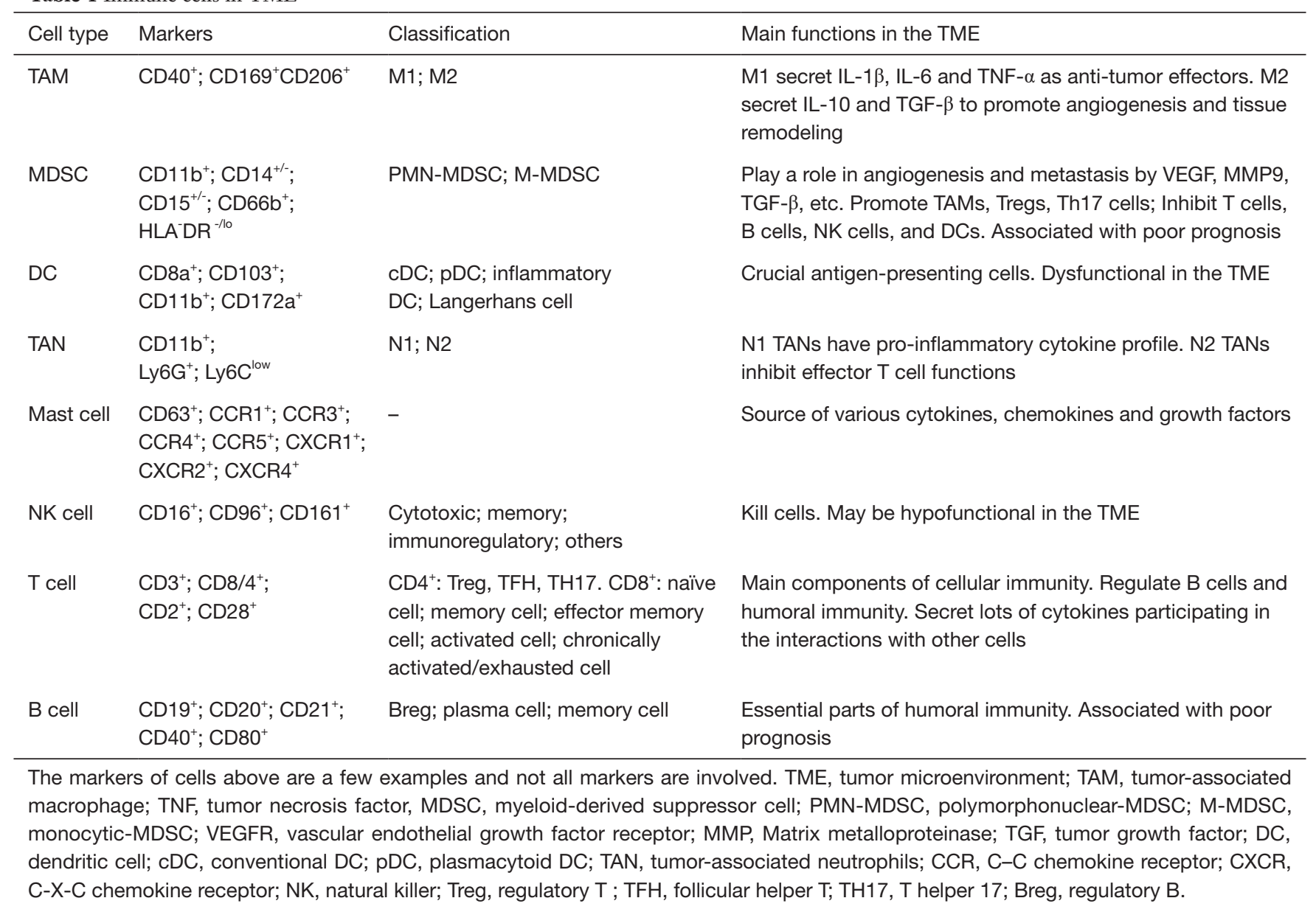

but infiltrate into cancer due to chronic inflammation (13). The way of accumulating and activating MDSCs requires two subsets of signals (14). First, growth factors produced by tumor cells and stroma, such as CSF, mediates the expansion of immature myeloid cells. Then, proinflammatory cytokines, including IL-6, IFN- $\gamma$, IL- $1 \beta$ and TNF- $\alpha$, define the immunosuppressive phenotype of the MDSCs. However, these two signals are necessary but not sufficient (15). It has been reported that MDSCs play an important role in immunosuppressive TME (16). MDSCs can promote tumor angiogenesis and metastasis by secreting cytokines including VEGF, MMP9 and TGF- $\beta$. Furthermore, MDSCs also promote TAMs, Tregs, Th17 cells while inhibiting T cells, B cells, NK cells, and DCs to induce the immunosuppressive effects. Importantly, the blood level of MDSCs was associated with the poor prognosis of breast cancer patients (17). The fraction of MDSCs in peripheral blood is about 10-fold higher in breast cancer patients than in healthy individuals (18). Furthermore, all-trans retinoic acid (ATRA) significantly decreased the frequency of circulating MDSCs compared to Ipilimumab treatment alone in advanced-stage melanoma patients (19), showing that targeting MDSCs could be promising in immunotherapy.

DCs are key antigen-presenting cells, which uptake, process and present antigenic peptides to $\mathrm{T}$ cells, leading to adaptive immune response and playing a pivotal role in TME. DCs contain several subsets, including conventional DCs (cDCs), plasmacytoid DCs (pDCs), inflammatory DCs and Langerhans cells (20). Among these DCs, CD103+ cDC1s seem to be the only $\mathrm{cDC}$ subset required to induce a cytotoxic $\mathrm{T}$-cell response against tumors upon blockade of the checkpoint ligand PD-L1 (21). However, due to the immunosuppressive nature of the TME, DCs around the tumor are often dysfunctional (22). Tumor-derived factors play dual roles in DCs functions. Cancer cells undergo cell 
death, resulting in the release of a host-derived damageassociated molecular pattern (DAMP). DAMP associated proteins, such as high-mobility group box 1 (HMGB1), heat-shock proteins (HSP), histones, the S100 family of proteins and serum amyloid $\mathrm{A}$, could be tumor-derived DC-activating factors. But VEGF, TGF- $\beta$, IL-10, IL8, IL-6, PSA and GDF-15 could be tumor-derived DCsuppressive factors $(23,24)$. Therefore, targeting DCs will be an interesting strategy to boost the specific T-cell response $(25,26)$. For instance, combined FLT3L and polyriboinosinic: polyribocytidylic acid therapy enhances tumor responses to BRAF and checkpoint blockade in melanoma through inducing expansion and activation of CD $103^{+}$DC progenitors in tumors (27).

The function of TANs is controversial, with both promoting or inhibiting tumor roles reported. Similar to TAMs, TANs also have two distinct functional phenotypes. TANs with the N1 phenotype are anti-tumor due to its pro-inflammatory cytokine profile producing hydrogen peroxide and being cytotoxic to tumor cells. N2 TANs, however, inhibit effector $\mathrm{T}$ cell functions by their high level of arginase 1 (ARG1) $(28,29)$. During tumor progression, the TAN composition changes dynamically, resulting in a switch from anti- to pro-tumor contribution (30). To be specific, the pro-tumor mechanisms may include tumor initiation, growth, angiogenesis, invasion and migration through various factors including VEGF, TNF $\alpha$, IL-17 and hydrogen peroxide (31). On the other hand, TANs can directly lysis and induce apoptosis or regulate $\mathrm{T}$ cell functions to indirectly suppress tumors (32). Tumor cells express a number of cytokines and chemokines including CXCL8, CXCL5 and CXCL6, which are involved in the recruitment of the TANs (33). It was reported that an enhanced level of TANs associated with poor prognosis of colorectal cancer patients (34). Therefore, understanding the function and status of TANs can help to modulate TME into the anti-cancer setting.

Mast cells, one of the hematopoietic cells, are "treasure house" of cytokines, chemokines, and growth factors (35). Thus, mast cells are important in inducing and maintaining immune response. Mast cells are recruited into TME early and play a significant role in tumor angiogenesis and remodeling in breast cancer $(36,37)$. Moreover, serine protease tryptase, one of the components of mast cells, could boost serum tryptase levels that correlate with poor prognosis in breast cancer patients (38). TNBC patients with high expression of annexin A1 also had more infiltration of mast cells and significantly shorter survival (39). Targeting mast cells are attractive therapeutic strategies for cancer treatment (40).

NK cells are genuine "natural killer" due to their spontaneous ability to kill cells through perforin- and granzyme-dependent mechanisms or by ligation of tumor-necrosis factor family (41). One of the most important functions of NK cells is the contribution to the immunosurveillance of cancer by innate lymphoid cells $(42,43)$. Nevertheless, due to the immunosuppressive microenvironment, NK cells in TME seem to be inert phenotype and hypofunctional (44). Therefore, re-activating NK cells in TME is an effective approach in treating cancer. For instance, using allogenic activated NK cells has been shown to be capable of killing cancer cells and even cancer stem cells (45). Notably, it was reported that NK cells mediated clinically relevant anti-metastatic effects (46). Releasing the inhibitory signals that limit NK cell function (KIR, NKG2A, TIGIT, TIM-3, LAG-3) are promising ways of NK cell-based immunotherapy (47). Nevertheless, NK cells have different functional subsets including cytotoxic, memory, immunoregulatory and licensedunlicensed subsets. Considering that the heterogeneity of NK cells is not fully understood yet, mobilizing the right subset of NK cells into TME remains a challenging task (48).

$\mathrm{T}$ cells are a predominant population of tumorinfiltration lymphocytes (TILs) and play a crucial role in coordinating multiple aspects of adaptive immunity (49). Antigens stimulate helper $\mathrm{T}$ cells to secrete cytokines (IL-2, 3, 4, 5, 6, 10, IFN- $\gamma$, TNF- $\alpha$, GM-CSF, etc.) as messengers. These cytokines can interact with other cells in the TME. For example, IL-4 can induce the differentiation of B cells into plasma cells and the secretion of antibodies. Cytotoxic T cells are the major effector cells in the adaptive immune system and can kill cancer cells through perforin and granzyme-mediated apoptosis. Single-cell sequencing showed that 9 distinct clusters of $\mathrm{T}$ cells were infiltrated in tumors. $\mathrm{CD}^{+}$clusters included regulatory $\mathrm{T}$ cells (Treg), follicular helper $\mathrm{T}(\mathrm{TFH})$ cells and T helper 17 cells (TH17) cells. $\mathrm{CD}^{+}$clusters included naive cells, memory cells, effector memory cells, activated cells, chronically activated/ exhausted cells (50). Furthermore, in breast cancer, a study has shown that $\mathrm{CD} 8^{+} \mathrm{CD} 103^{+}$tissue-resident memory $\mathrm{T}$ cells contribute to the immunosurveillance of cancer (51). Additionally, T cells with exhausted phenotype could not function well in the TME, so how to transform exhausted $\mathrm{T}$ cells to activated $\mathrm{T}$ cells will be a promising target for clinical therapy $(52,53)$. As an example, using monoclonal antibodies to block cell surface markers, such as CTLA-4, 
Table 2 Stromal cells in the TME

\begin{tabular}{|c|c|c|c|}
\hline Cell type & Markers & Classification & Main functions in the TME \\
\hline $\begin{array}{l}\text { Endothelial } \\
\text { cell }\end{array}$ & $\begin{array}{l}\mathrm{CD}^{+} 1^{+} ; \mathrm{CD}^{+} 4^{+} ; \mathrm{CD} 105^{+} \\
\mathrm{CD}^{+} 06^{+} ; \mathrm{CD} 144^{+}\end{array}$ & $\begin{array}{l}\text { Vascular endothelial cell. Lymphatic } \\
\text { endothelial cell }\end{array}$ & $\begin{array}{l}\text { Help with angiogenesis, tumor metastasis and drug } \\
\text { resistance }\end{array}$ \\
\hline Adipocyte & $\begin{array}{l}\text { Adipokines: adiponectin, } \\
\text { resistin; leptin }\end{array}$ & - & $\begin{array}{l}\text { Interact with tumor cells in tumor initiation, tumor } \\
\text { progression, invasion, and metastasis }\end{array}$ \\
\hline MSC & $\begin{array}{l}\mathrm{CD} 44^{+} ; \mathrm{CD}^{+} 3^{+} ; \mathrm{CD}^{+} 0^{+} \\
\mathrm{CD} 105^{+} ; \mathrm{DDR}^{+}\end{array}$ & $\begin{array}{l}\text { Can differentiate to various cells like } \\
\text { adipocyte, osteocyte, chondrocyte, etc. }\end{array}$ & $\begin{array}{l}\text { Communicate with tumor cells through paracrine } \\
\text { signaling. MSC-derived exosomes are important in } \\
\text { tumorigenesis, angiogenesis, and metastasis }\end{array}$ \\
\hline
\end{tabular}

The markers of cells above are a few examples and not all markers are involved. CAF, cancer-associated fibroblast; $\alpha$ SMA, $\alpha$-smooth muscle actin; FAP, fibroblast activation protein; PDGFvR, platelet-derived growth factor receptor; MSC, mesenchymal stem cell.

PD-1 or PD-L1, shows great potential to reinvigorate exhausted $T$ cells (54). In addition, chimeric antigen receptor (CAR) $\mathrm{T}$ cell therapy, designed to reinforce $\mathrm{T}$ cell activity, is being tested in solid tumors including breast cancer (55). Yet, tumor antigen escape and downregulation could impact the duration of CAR-T cells and could be a potential target (56).

Comparing to the well-established role of $\mathrm{T}$ cells in TME, the contributions of B cells to cancer are less studied. $B$ cells and their effect cells, plasma cells, are essential parts of humoral immunity (57). BCR-related kinases, such as BTK, also make a difference in the TME of solid tumors (58). Regulatory B cells (B-regs) are capable to harness T-cell responses and promote tumor growth (57). $\mathrm{B}$ cells in the TME are a heterogeneous population with diverse functions, so the role of $\mathrm{B}$ cells is also dual (59). The balance of pro- and anti-tumor effects is dynamic (60). It was shown that an enhanced level of B cells in breast cancer was associated with poor prognostic biomarkers including negative estrogen receptor (ER) status, IL-10 secretion and PD-L1 expression and high-grade tumor $(61,62)$. In contrast, other studies indicated B cells play a beneficial role in the majority of cancers (63). As a result, further studies are required to figure out the correlation between the subclasses of B cells and different subtypes of breast cancer.

\section{Stromal cells}

In addition to the above immune cells, stromal cells, including cancer-associated fibroblasts (CAFs), endothelial cells, adipocytes and pericytes, also play an essential role in TME. These cells work together to establish an immunosuppressive, angiogenetic TME (64). Moreover, factors secreted by these stromal cells have a great influence on tumor progression and metastasis $(65,66)$ (Table 2).

Among all the stromal cells infiltrating in the TME, CAFs have received the most attention due to the critical contribution to cancer progression. The functions of CAFs are related to immune suppression, ECM remodeling and the secretion of pro-inflammatory and pro-metastatic cytokines, enzymes and microRNAs (67). CAFs shape extracellular matrix (ECM) structure by secreting collagen and other fibrous macromolecules as well as releasing proteolytic enzymes. Thus, CAFs are essential in maintaining cell mobility (68). It is known that activated fibroblasts regulate tumor progression, and markers like fibroblast specific protein 1 (FSP1 or S100A4), vimentin, $\alpha$ SMA, FAP, PDGFR $\alpha / \beta$, DDR2 can identify the activated CAFs (69). Similar to the immune cells in TME, the functions of the CAFs also have two sides. Pro-tumoral CAF subsets are associated with tumorigenesis, tumor angiogenesis, tumor metastasis and drug resistance. In contrast, anti-tumoral CAF subsets can be tumor-suppressive (70). For instance, CD146 ${ }^{+}$CAFs sustain tamoxifen sensitivity in $\mathrm{ER}^{+}$breast cancer (71). Therefore, with the fact that CAFs are a heterogeneous cell population of multiple origins, therapies targeting CAFs must be specific (72). It was shown recently that a subset 
of $\mathrm{CD} 10^{+} \mathrm{GPR} 77^{+} \mathrm{CAFs}$ promoted cancer formation and chemoresistance by sustaining cancer stem cells (73). Smoothened (SMO) inhibitors (itraconazole and vismodegib), histone deacetylase (HDAC) inhibitors, which target CAFs or their precursors, have been testing in clinical trials $(74,75)$. The prospect of CAFs-associated therapies seems promising and further investigations are needed.

Endothelial cells comprise vascular endothelial cells and lymphatic endothelial cells. The interactions between cancer cells and endothelial cells change the phenotype of the endothelial cells by soluble factors (VEGF), adhesion receptors, gap junctions and exosomes $(64,76)$. Through these ways, cancer cells communicate with endothelial cells and promote angiogenesis. As for lymphatic endothelial cells, they can promote metastasis under the regulation of cancer cells. Cancer cells activated the IL-6/STAT pathway inducing CCL5 expression in the lymphatic endothelial cells (77). The crosstalk between endothelial cells and cancer cells can be therapeutic targets against tumor progression and drug resistance (78).

Pericytes are derived from mesenchymal cells and maintain the stability of capillaries. Also, pericytes can interact with endothelial cells during vessel formation. The markers of pericytes include PDGFR- $\beta$, NG2, CD13, $\alpha \mathrm{SMA}$ and desmin (79). Aberrant pericytes result in aberrant tumor vessels which contribute to the tumor progression and drug resistance (80). Strikingly, pericytes also participate in the formation of the premetastatic niche (81). Therefore, maintain normal pericytes could be a promising negative regulator of tumor metastasis.

Mesenchymal stem cells (MSCs) are multipotent stromal cells, which means they can differentiate into other mesenchymal cells like adipocytes in TME. The common surface protein expression pattern of the MSCs is $\mathrm{CD} 44^{+} \mathrm{CD} 73^{+} \mathrm{CD} 90^{+} \mathrm{CD} 105^{+}(66)$. Collagen receptor tyrosine kinases DDR2, activated by fibrillar collagen, is expressed specifically in the MSCs. It was reported that MSC-induced DDR2 mediated stromal-breast cancer interactions and metastasis growth (82). MSCs may interact with tumor cells through paracrine activity (83). Growing studies suggest that MSC-derived exosomes function as mediators in the tumor niche and play several roles in tumorigenesis, angiogenesis, and metastasis (84).

Researches on adipocytes are scanty compared to other cells in the TME. Adipocytes, take up 7\% to $56 \%$ in breast tissue, closely interact with breast cancer cells in tumor initiation, tumor progression, invasion, and metastasis (85). Adipocytes involved in tumor progression are also called cancer-associated adipocytes (CAAs). It was shown that mature adipocytes secret IGFBP-2 promoting tumor metastasis in breast cancer cells (86). Adipokines (adiponectin, resistin, and leptin) secreted by adipocytes were associated with poor prognosis of breast cancer patients (87). Moreover, obesity was shown to be a driver of therapeutic resistance in breast cancer cells (88). Hence, exercise and other therapies targeting adiposity and adipokines may have potential in improving outcomes of breast cancer $(89,90)$.

\section{Concluding remarks and perspectives}

TMEs compromise malignant cells and non-malignant cells. Non-malignant cells in TME often function as accomplices to cancer cells. Therefore, cancer treatments should also target these non-malignant cells. More importantly, these non-malignant cells are not genetically "bad" (mutated), but are pro-tumor because of epigenetic dysregulation. Inducing these flexible non-malignant cells into the antitumor phenotype is a very attractive and successful strategy, with much room to be improved. It is worth noting that understanding the complex interactions among different cells in TME can lead to more selective or precise cancer therapy. Novel techniques such as single cell sequencing provide plenty of chances to understand the components in the TME thoroughly. These findings may shed light on the immune regulatory mechanism in the TME, and eventually lead to more effective cancer treatment.

\section{Acknowledgments}

Funding: This work was funded by Natural Science Foundation of China grants (81872141 and 81630074), Guangzhou Science and Technology plan key projects (201804020076).

\section{Footnote}

Conflicts of Interest: Both authors have completed the ICMJE uniform disclosure form (available at http://dx.doi.org/10.21037/tbcr.2020.03.07). The authors have no conflicts of interest to declare.

Ethical Statement: The authors are accountable for all 
aspects of the work in ensuring that questions related to the accuracy or integrity of any part of the work are appropriately investigated and resolved.

Open Access Statement: This is an Open Access article distributed in accordance with the Creative Commons Attribution-NonCommercial-NoDerivs 4.0 International License (CC BY-NC-ND 4.0), which permits the noncommercial replication and distribution of the article with the strict proviso that no changes or edits are made and the original work is properly cited (including links to both the formal publication through the relevant DOI and the license). See: https://creativecommons.org/licenses/by-ncnd/4.0/.

\section{References}

1. Yuan Y, Jiang YC, Sun CK, et al. Role of the tumor microenvironment in tumor progression and the clinical applications. Oncol Rep 2016;35:2499-515.

2. Wang M, Zhao J, Zhang L, et al. Role of tumor microenvironment in tumorigenesis. J Cancer 2017;8:761-73.

3. Balkwill FR, Capasso M, Hagemann T. The tumor microenvironment at a glance. J Cell Sci 2012;125:5591-6.

4. Newman AM, Liu CL, Green MR, et al. Robust enumeration of cell subsets from tissue expression profiles. Nat Methods 2015;12:453-7.

5. Aran D, Hu Z, Butte AJ. xCell: digitally portraying the tissue cellular heterogeneity landscape. Genome Biol 2017;18:220.

6. Binnewies M, Roberts EW, Kersten K, et al. Understanding the tumor immune microenvironment (TIME) for effective therapy. Nat Med 2018;24:541-50.

7. Mantovani A, Sica A. Macrophages, innate immunity and cancer: balance, tolerance, and diversity. Curr Opin Immunol 2010;22:231-7.

8. Ngambenjawong C, Gustafson HH, Pun SH. Progress in tumor-associated macrophage (TAM)-targeted therapeutics. Adv Drug Deliv Rev 2017;114:206-21.

9. Quail DF, Joyce JA. Molecular Pathways: Deciphering Mechanisms of Resistance to Macrophage-Targeted Therapies. Clin Cancer Res 2017;23:876-84.

10. Wilson JL, Nagele T, Linke $M$, et al. Inverse Data-Driven Modeling and Multiomics Analysis Reveals Phgdh as a Metabolic Checkpoint of Macrophage Polarization and Proliferation. Cell Rep 2020;30:1542-52 e1547.

11. Veglia F, Perego M, Gabrilovich D. Myeloid-derived suppressor cells coming of age. Nature Immunology 2018;19:108-19.

12. Bronte V, Brandau S, Chen SH, et al. Recommendations for myeloid-derived suppressor cell nomenclature and characterization standards. Nat Commun 2016;7:12150.

13. Tesi RJ. MDSC; the Most Important Cell You Have Never Heard Of. Trends Pharmacol Sci 2019;40:4-7.

14. Marvel D, Gabrilovich DI. Myeloid-derived suppressor cells in the tumor microenvironment: expect the unexpected. J Clin Invest 2015;125:3356-64.

15. Ortiz ML, Kumar V, Martner A, et al. Immature myeloid cells directly contribute to skin tumor development by recruiting IL-17-producing CD4+ T cells. J Exp Med 2015;212:351-67.

16. Yin Z, Li C, Wang J, et al. Myeloid-derived suppressor cells: Roles in the tumor microenvironment and tumor radiotherapy. Int J Cancer 2019;144:933-46.

17. Dias AS, Almeida CR, Helguero LA, et al. Metabolic crosstalk in the breast cancer microenvironment. Eur J Cancer 2019;121:154-71.

18. Safarzadeh E, Hashemzadeh S, Duijf PHG, et al. Circulating myeloid-derived suppressor cells: An independent prognostic factor in patients with breast cancer. J Cell Physiol 2019;234:3515-25.

19. Tobin RP, Jordan KR, Robinson WA, et al. Targeting myeloid-derived suppressor cells using all-trans retinoic acid in melanoma patients treated with Ipilimumab. Int Immunopharmacol 2018;63:282-91.

20. Qian C, Cao X. Dendritic cells in the regulation of immunity and inflammation. Semin Immunol 2018;35:3-11.

21. Gardner A, Ruffell B. Dendritic Cells and Cancer Immunity. Trends Immunol 2016;37:855-65.

22. Veglia F, Gabrilovich DI. Dendritic cells in cancer: the role revisited. Curr Opin Immunol 2017;45:43-51.

23. Zong J, Keskinov AA, Shurin GV, et al. Tumor-derived factors modulating dendritic cell function. Cancer Immunol Immunother 2016;65:821-33.

24. Tang M, Diao J, Cattral MS. Molecular mechanisms involved in dendritic cell dysfunction in cancer. Cell Mol Life Sci 2017;74:761-76.

25. Szaryńska M, Olejniczak A, Kobiela J, et al. Cancer stem cells as targets for DC-based immunotherapy of colorectal cancer. Sci Rep 2018;8:12042.

26. Conejo-Garcia JR, Rutkowski MR, Cubillos-Ruiz JR. State-of-the-art of regulatory dendritic cells in cancer. Pharmacol Ther 2016;164:97-104.

27. Salmon H, Idoyaga J, Rahman A, et al. Expansion and Activation of CD103(+) Dendritic Cell Progenitors at the 
Tumor Site Enhances Tumor Responses to Therapeutic PD-L1 and BRAF Inhibition. Immunity 2016;44:924-38.

28. Hagerling C, Werb Z. Neutrophils: Critical components in experimental animal models of cancer. Semin Immunol 2016;28:197-204.

29. Shaul ME, Levy L, Sun J, et al. Tumor-associated neutrophils display a distinct N1 profile following TGFbeta modulation: A transcriptomics analysis of provs. antitumor TANs. Oncoimmunology 2016;5:e1232221.

30. Sagiv JY, Michaeli J, Assi S, et al. Phenotypic diversity and plasticity in circulating neutrophil subpopulations in cancer. Cell Rep 2015;10:562-73.

31. Powell DR, Huttenlocher A. Neutrophils in the Tumor Microenvironment. Trends Immunol 2016;37:41-52.

32. Uribe-Querol E, Rosales C. Neutrophils in Cancer: Two Sides of the Same Coin. J Immunol Res 2015;2015:983698.

33. Del Prete A, Schioppa T, Tiberio L, et al. Leukocyte trafficking in tumor microenvironment. Curr Opin Pharmacol 2017;35:40-7.

34. Galdiero MR, Bianchi P, Grizzi F, et al. Occurrence and significance of tumor-associated neutrophils in patients with colorectal cancer. Int J Cancer 2016;139:446-56.

35. Mukai K, Tsai M, Saito H, et al. Mast cells as sources of cytokines, chemokines, and growth factors. Immunol Rev 2018;282:121-50.

36. Cimpean AM, Tamma R, Ruggieri S, et al. Mast cells in breast cancer angiogenesis. Crit Rev Oncol Hematol 2017;115:23-6.

37. Sammarco G, Varricchi G, Ferraro V, et al. Mast Cells, Angiogenesis and Lymphangiogenesis in Human Gastric Cancer. Int J Mol Sci 2019. doi: 10.3390/ijms20092106.

38. Aponte-López A, Fuentes-Panana EM, Cortes-Munoz D, et al. Mast Cell, the Neglected Member of the Tumor Microenvironment: Role in Breast Cancer. J Immunol Res 2018;2018:2584243.

39. Okano M, Oshi M, Butash AL, et al. Triple-Negative Breast Cancer with High Levels of Annexin A1 Expression Is Associated with Mast Cell Infiltration, Inflammation, and Angiogenesis. Int J Mol Sci 2019. doi: 10.3390/ ijms20174197.

40. Ribatti D. Mast cells as therapeutic target in cancer. Eur J Pharmacol 2016;778:152-7.

41. Li Y, Yin J, Li T, et al. NK cell-based cancer immunotherapy: from basic biology to clinical application. Sci China Life Sci 2015;58:1233-45.

42. Chiossone L, Dumas PY, Vienne M, et al. Natural killer cells and other innate lymphoid cells in cancer. Nat Rev Immunol 2018;18:671-88.
43. Morvan MG, Lanier LL. NK cells and cancer: you can teach innate cells new tricks. Nat Rev Cancer 2016;16:7-19.

44. Del Zotto G, Marcenaro E, Vacca P, et al. Markers and function of human NK cells in normal and pathological conditions. Cytometry B Clin Cytom 2017;92:100-14.

45. Ferreira-Teixeira M, Paiva-Oliveira D, Parada B, et al. Natural killer cell-based adoptive immunotherapy eradicates and drives differentiation of chemoresistant bladder cancer stem-like cells. BMC Med 2016;14:163.

46. López-Soto A, Gonzalez S, Smyth MJ, et al. Control of Metastasis by NK Cells. Cancer Cell 2017;32:135-54.

47. Sanchez-Correa B, Lopez-Sejas N, Duran E, et al. Modulation of NK cells with checkpoint inhibitors in the context of cancer immunotherapy. Cancer Immunol Immunother 2019;68:861-70.

48. Guillerey C, Huntington ND, Smyth MJ. Targeting natural killer cells in cancer immunotherapy. Nat Immunol 2016;17:1025-36.

49. Kumar BV, Connors TJ, Farber DL. Human T Cell Development, Localization, and Function throughout Life. Immunity 2018;48:202-13.

50. Yost KE, Satpathy AT, Wells DK, et al. Clonal replacement of tumor-specific T cells following PD-1 blockade. Nat Med 2019;25:1251-9.

51. Savas P, Virassamy B, Ye C, et al. Single-cell profiling of breast cancer $\mathrm{T}$ cells reveals a tissue-resident memory subset associated with improved prognosis. Nat Med 2018;24:986-93.

52. Chung W, Eum HH, Lee HO, et al. Single-cell RNAseq enables comprehensive tumour and immune cell profiling in primary breast cancer. Nat Commun 2017;8:15081.

53. Thommen DS, Schumacher TN. T Cell Dysfunction in Cancer. Cancer Cell 2018;33:547-62.

54. Catakovic K, Klieser E, Neureiter D, et al. T cell exhaustion: from pathophysiological basics to tumor immunotherapy. Cell Commun Signal 2017;15:1.

55. Newick K, O'Brien S, Moon E, et al. CAR T Cell Therapy for Solid Tumors. Annu Rev Med 2017;68:139-52.

56. Majzner RG, Mackall CL. Tumor Antigen Escape from CAR T-cell Therapy. Cancer Discov 2018;8:1219-26.

57. Sarvaria A, Madrigal JA, Saudemont A. B cell regulation in cancer and anti-tumor immunity. Cell Mol Immunol 2017;14:662-74.

58. Burger JA, Wiestner A. Targeting B cell receptor signalling in cancer: preclinical and clinical advances. Nat Rev Cancer 2018;18:148-67.

59. Zhang Z, Zhu Y, Wang Z, et al. Yin-yang effect of tumor 
infiltrating B cells in breast cancer: From mechanism to immunotherapy. Cancer Lett 2017;393:1-7.

60. Tsou P, Katayama H, Ostrin EJ, et al. The Emerging Role of B Cells in Tumor Immunity. Cancer Res 2016;76:5597-601.

61. Guan H, Lan Y, Wan Y, et al. PD-L1 mediated the differentiation of tumor-infiltrating CD19(+) B lymphocytes and $\mathrm{T}$ cells in Invasive breast cancer. Oncoimmunology 2015;5:e1075112.

62. Shen M, Wang J, Ren X. New Insights into TumorInfiltrating B Lymphocytes in Breast Cancer: Clinical Impacts and Regulatory Mechanisms. Front Immunol 2018;9:470.

63. Wouters MCA, Nelson BH. Prognostic Significance of Tumor-Infiltrating B Cells and Plasma Cells in Human Cancer. Clinical Cancer Research 2018;24:6125-35.

64. De Palma M, Biziato D, Petrova TV. Microenvironmental regulation of tumour angiogenesis. Nat Rev Cancer 2017;17:457-74.

65. Guo S, Deng CX. Effect of Stromal Cells in Tumor Microenvironment on Metastasis Initiation. Int J Biol Sci 2018;14:2083-93.

66. Ridge SM, Sullivan FJ, Glynn SA. Mesenchymal stem cells: key players in cancer progression. Mol Cancer 2017;16:31.

67. Wang Z, Tan Y, Yu W, et al. Small role with big impact: miRNAs as communicators in the cross-talk between cancer-associated fibroblasts and cancer cells. Int J Biol Sci 2017;13:339-48.

68. Valkenburg KC, de Groot AE, Pienta KJ. Targeting the tumour stroma to improve cancer therapy. Nat Rev Clin Oncol 2018;15:366-81.

69. Kalluri R. The biology and function of fibroblasts in cancer. Nat Rev Cancer 2016;16:582-98.

70. Chen X, Song E. Turning foes to friends: targeting cancer-associated fibroblasts. Nat Rev Drug Discov 2019;18:99-115.

71. Brechbuhl HM, Finlay-Schultz J, Yamamoto TM, et al. Fibroblast Subtypes Regulate Responsiveness of Luminal Breast Cancer to Estrogen. Clin Cancer Res 2017;23:1710-21.

72. Lynch MD, Watt FM. Fibroblast heterogeneity: implications for human disease. J Clin Invest 2018;128:26-35.

73. Su S, Chen J, Yao H, et al. CD10(+)GPR77(+) CancerAssociated Fibroblasts Promote Cancer Formation and Chemoresistance by Sustaining Cancer Stemness. Cell 2018;172:841-856.e16.

74. Younes A, Berdeja JG, Patel MR, et al. Safety, tolerability, and preliminary activity of CUDC-907, a first-in-class, oral, dual inhibitor of HDAC and PI3K, in patients with relapsed or refractory lymphoma or multiple myeloma: an open-label, dose-escalation, phase 1 trial. The Lancet Oncology 2016;17:622-31.

75. Kim DJ, Kim J, Spaunhurst K, et al. Open-label, exploratory phase II trial of oral itraconazole for the treatment of basal cell carcinoma. J Clin Oncol 2014;32:745-51.

76. Lopes-Bastos BM, Jiang WG, Cai J. Tumour-Endothelial Cell Communications: Important and Indispensable Mediators of Tumour Angiogenesis. Anticancer Res 2016;36:1119-26.

77. Lee E, Fertig EJ, Jin K, et al. Breast cancer cells condition lymphatic endothelial cells within pre-metastatic niches to promote metastasis. Nat Commun 2014;5:4715.

78. Choi H, Moon A. Crosstalk between cancer cells and endothelial cells: implications for tumor progression and intervention. Arch Pharm Res 2018;41:711-24.

79. Armulik A, Genove G, Betsholtz C. Pericytes: developmental, physiological, and pathological perspectives, problems, and promises. Dev Cell 2011;21:193-215.

80. Caporali A, Martello A, Miscianinov V, et al. Contribution of pericyte paracrine regulation of the endothelium to angiogenesis. Pharmacol Ther 2017;171:56-64.

81. Paiva AE, Lousado L, Guerra DAP, et al. Pericytes in the Premetastatic Niche. Cancer Research 2018;78:2779-86.

82. Gonzalez ME, Martin EE, Anwar T, et al. Mesenchymal Stem Cell-Induced DDR2 Mediates Stromal-Breast Cancer Interactions and Metastasis Growth. Cell Rep 2017;18:1215-28.

83. Spees JL, Lee RH, Gregory CA. Mechanisms of mesenchymal stem/stromal cell function. Stem Cell Res Ther 2016;7:125.

84. Vakhshiteh F, Atyabi F, Ostad SN. Mesenchymal stem cell exosomes: a two-edged sword in cancer therapy. Int J Nanomedicine 2019;14:2847-59.

85. Choi J, Cha YJ, Koo JS. Adipocyte biology in breast cancer: From silent bystander to active facilitator. Prog Lipid Res 2018;69:11-20.

86. Wang C, Gao C, Meng K, et al. Human adipocytes stimulate invasion of breast cancer MCF-7 cells by secreting IGFBP-2. PLoS One 2015;10:e0119348.

87. Li J, Han X. Adipocytokines and breast cancer. Curr Probl Cancer 2018;42:208-14.

88. Hoy AJ, Balaban S, Saunders DN. Adipocyte-Tumor Cell Metabolic Crosstalk in Breast Cancer. Trends Mol Med 
2017;23:381-92.

89. Pudkasam S, Tangalakis K, Chinlumprasert N, et al. Breast cancer and exercise: The role of adiposity and immune markers. Maturitas 2017;105:16-22.

doi: $10.21037 /$ tbcr.2020.03.07

Cite this article as: Wang Q, Liu Q. Tumor microenvironment and future targets of immunotherapy in breast cancer. Transl Breast Cancer Res 2020;1:6.
90. Cha YJ, Koo JS. Adipokines as therapeutic targets in breast cancer treatment. Expert Opin Ther Targets 2018;22:941-53. 